\title{
Successful Bone Grafting via Masquelet Technique Around a Locked Antibiotic Nail for Tibial Shaft Fracture with Polymicrobial Infection
}

\author{
Tyler Snoap, Jessica Hart, and Jason Roberts ${ }^{2}$
}

\begin{abstract}
Background: The clinical course of a 24-year-old male who sustained an open crush injury to his left tibia is detailed.

Case Presentation: The patient presented several treatment challenges for the orthopedic surgery team as well as the infectious disease service. He had a severe polymicrobial infection, a fractured long bone, bone loss, and an unstable soft tissue envelope.

Conclusion: The authors describe their multi-system team approach to address each of these issues, which resulted in eradication of infection and a stable, weight-bearing limb.
\end{abstract}

Keywords: infection; masquelet; tibia; trauma

$\mathbf{T}$ HE TREATMENT of infected, diaphyseal fractures with bone loss poses a challenge to the treating physicians. This can be even more complicated when orthopedic implants have been inserted previously prior to the exhibition of infection. There is a balance that must take place between providing fracture stability while eradicating infection. In certain situations, one may choose to address one issue at a time. Retention of the orthopedic implant while suppressing the infection until union is one option. Removal of orthopedic implants after achieving union often then takes place at a later procedure. Alternatively, orthopedic implants can be removed at the expense of stability with the aim of eradicating infection and providing stability at a future procedure. In either scenario, orthopedic implants are often removed because pathogens can form glycocalyces and biofilms making systemic antibiotic delivery less effective. For this reason, local antibiotic delivery via eluting antibiotic cement has gained popularity. This can take the form of antibiotic beads, cement spacers, or cement rods. The rendering of an antibiotic rod or nail has been described by several authors [1-3]. Thonse and Conway [3] have one of the largest series of using antibiotic nails for treatment of infected non-unions with 52 patients. A select group in their series did require additional surgery after placement of the antibiotic nail, of which one of these procedures involved bone grafting. The timing and methodology of the bone grafting in the presence of the antibiotic nail was not addressed in their article [3].

We present a case report with the methodology used to address a severe polymicrobial infected, fractured long bone with associated bone loss. After removal of initial hardware and serial debridements, a locked, metallic antibiotic nail was placed. For six weeks the patient received tailored, intravenous antibiotic agents per the infectious disease service. Once clinical signs of infection subsided, the fracture site was bone grafted adjacent to the retained metallic antibiotic nail using the masquelet technique and exchange nailing was not performed. The patient went on to union and maintained a functional limb.

\section{Case Presention}

A 24-year-old otherwise healthy male sustained an open crush injury to his left tibia while operating heavy machinery at a meat processing plant. He presented with a Gustilo and

\footnotetext{
${ }^{1}$ Western Michigan University Homer Stryker MD School of Medicine, Department of Orthopaedics, Kalamazoo, Michigan.

${ }^{2}$ Bronson Orthopedic Trauma, Kalamazoo, Michigan.

(C) Tyler Snoap et al. 2017; Published by Mary Ann Liebert, Inc. This Open Access article is distributed under the terms of the Creative Commons License (http://creativecommons.org/licenses/by/4.0), which permits unrestricted use, distribution, and reproduction in any medium, provided the original work is properly credited.
} 
Anderson grade IIIB tibial shaft fracture shown in Figure 1. On the day of injury, he underwent irrigation and debridement until the remaining muscle and soft tissue envelop appeared free of contamination. The wound bed at that time looked appropriate for definitive fixation and thus an intramedullary tibial nail was implanted. A wound vac was placed over a large medial soft tissue wound with plans for flap coverage by plastic surgery at a later date. Plans for future skin coverage changed shortly thereafter when an aggressive, polymicrobial infection just five days after the index procedure developed. Intra-operative cultures at the time of irrigation and debridement were positive for multiple organisms including Escherichia coli, Enterobacter cloacae, Enterococcus faecalis, Proteus mirabilis, Morganella morgana, Bacteroides fragilis, streptococcus, and staphylococcus. The polymicrobial nature of his infection was attributed to his employment at a meat processing plant with microscopic contamination remaining after the intial debridement. Amputation was considered at this juncture given his severe polymicrobial infection in the presence of a concomitant bone and soft tissue injury. Limb salvage was also presented as an option; no guarentees were made in regards to the success of this route of treatment. The patient elected to proceed with the latter treatment option.

The process of limb salvage began with radical debridement of the soft tissue and bone with removal of the implanted tibial nail. This was followed by multiple debridements and temporary antibiotic cement spacers using vancomycin and tobramycin to control the infection and fill an $8-\mathrm{cm}$ cortical gap in the tibia. The temporary antibiotic spacers acted to fill the dead space adjacent to the viable bone and also allowed for elution of local antibiotics. A series of eight debridement procedures in the operating room were performed every 48-72 hours that included flushing of the tibial canal and exchange of the antibiotic block spacer. The irrigant chosen was irrisept, a low concentration of chlorhexidine gluconate that was administered using low pressure lavage to minimize irritation of the soft tissues. Systemic antibiotic agents were administered simulataneously during this time based on recommendations from the infectious disease service. Vancomycin and meropenem were administered via a peripherally inserted central catheter (PICC) line for approximately six weeks. Roughly three weeks after his initial injury, the wound bed looked amenable to placement of more rigid fixation. A metallic, antibiotic locked nail was placed with a surrounding cement block spacer to fill the bony defect. The technique used to fashion the locked, metallic nail has been previously described by Hake et al [2].

Plastic surgery was able to perform flap coverage using the soleus and gastrocnemius muscles over the ensuing weeks. Roughly five months after the original injury when inflammatory laboratory tests returned to baseline, the patient underwent Reamer-irrigator-aspirator (RIA-Depuy Synthes) bone grafting using the ipsilateral femur as the donor site. The antibiotic spacer was removed at the time of the procedure and the Masquelet-induced membrane technique was used to fill the bony defect (Fig. 2) [4]. The metallic antibiotic nail was retained at this procedure. The patient was followed closely in the clinic and eventually went on to an infectionfree union shown in Figure 3. He was seen most recently at his 2.5-year clinic visit ambulating without an assistive device, using occasional ibuprofen for pain. He no longer taking antibiotic agents and mechanical stability to his leg has been restored.

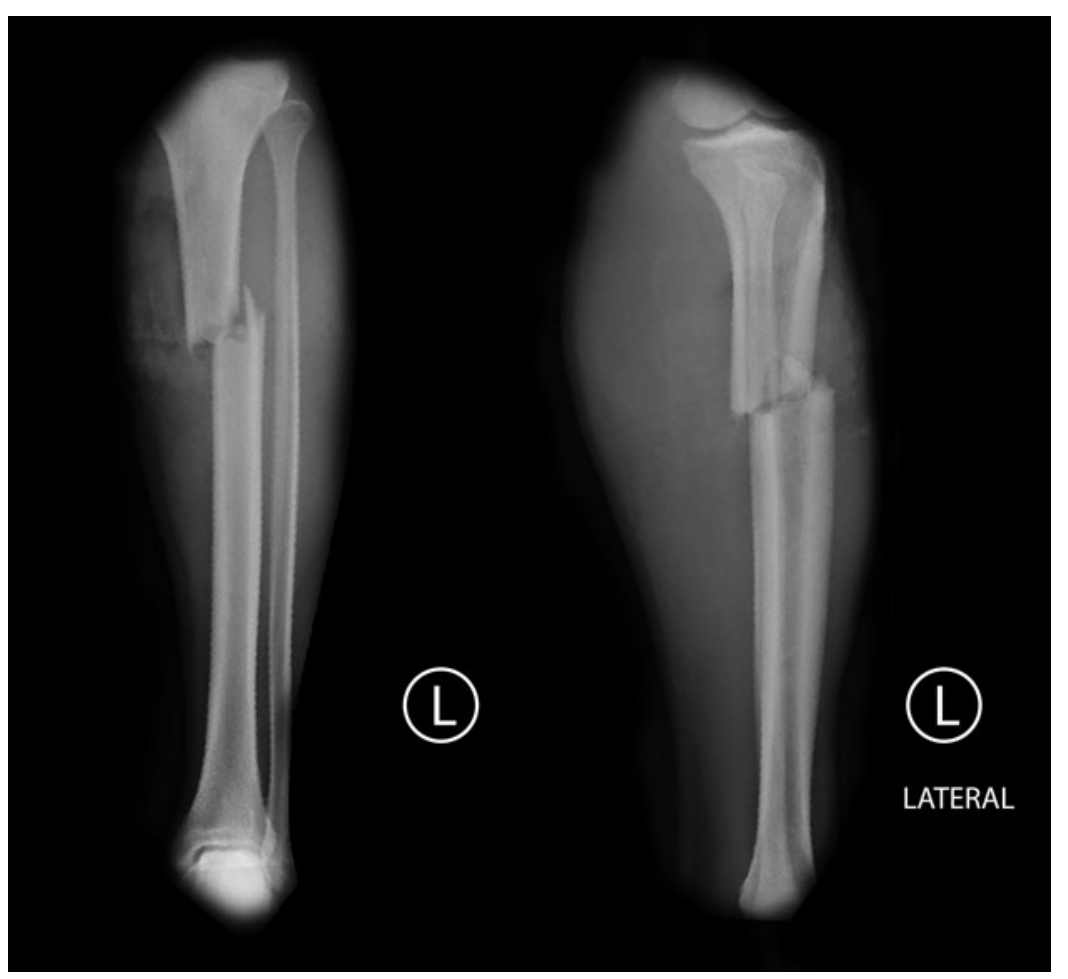

FIG. 1. Anteroposterior and lateral injury film demonstrating a transverse tibial shaft fracture with evidence of medial soft tissue injury. 




FIG. 2. Anteroposterior and lateral radiograph demonstrating the metallic antibiotic locked nail after performing the Masquelet technique and placing autologous bone graft. Note the large bony defect.

\section{Discussion and Literature Review}

The management of a diaphyseal fracture with bone loss in the setting of infection poses a therapeutic challenge to the treating physicians. The goal of treatment is to eradicate the infection and stabilize the fracture site to allow union. There are many different treatment methods to address this enigma, all based on certain key concepts. First, orthopedic implants generally need to be removed because they can impair the ability to eradicate infection. Second, adding stability to the



FIG. 3. Anteroposterior and lateral radiographs at 29-month follow-up radiograph demonstrating union of the fracture site with the retained metallic antibiotic nail. 
soft tissue envelop is important. The exact reasons why stability aids in clearing infection is likely multi-factorial. Stability of the bone provides stability to the soft tissue, in turn there is less irritation, inflammation, and hematoma propagation. Perhaps most important is the stability necessary for revascularization to take place [5]. Third, careful management of the dead space in the long bone canal should be addressed along with local antibiotic administration. Unlike most forms of osteomyelitis, intra-medullary bone osteomyelitis generally does not contain a sequestrum or dead bone material. However, even in the absence of infarcted bone, the intra-medullary canal acts like an avascular dead space that is not collapsible. This space needs to be filled and in doing so there is less likely a risk of recurrence of infection after bone debridement.

Antibiotic nails address many of the issues mentioned above. Paley and Herzenberg [1] published their series of nine patients who were treated in this fashion. They reported $100 \%$ clearance of infection in their study [1]. Thonse and Conway [3] in their series of antibiotic metallic nails described the necessity of a secondary surgery after nail placement in $27 \%$ of cases. They include bone grafting as one of these procedures but do not address their specific methodology such as graft type or donor site if autograft was selected [3].

We present the results of a technique used to treat a traumatic open tibial fracture with associated bone loss due to radical debridement for infection. The first procedure performed was removal of orthopedic implants and a thorough debridement. After serial debridements every 48-72 hours, we used the technique described by Hake et al. [2] to implant a locked, metallic antibiotic nail. Systemic antibiotic agents were delivered based on the infectious disease service recommendations. Once the signs and symptoms of infection cleared we used autologous bone graft around the metallic antibiotic nail via the Masquelet technique to augment the fracture biology. By leaving the antibiotic nail in place, there is theoretically less of a chance of autograft resorption or development of central necrosis around the large area of autograft [4].

There are some potential risks to this technique. First, one must be certain that the infection has cleared because placing an autologous bone graft into an infected non-union site could facilitate further spread of the infection and add another nidus. Second, there is in vitro theoretical evidence that bacteria can persist on antibiotic-impregnated cement [6]. The clinical significance of this is unknown as Moussa et al. [7] published a series of asymptomatic patients who had cultures taken from wounds and orthopedic implants at the time of hardware removal. Although roughly half of the patient's had positive culture results, none presented clinically with infection and none were treated with antibiotic agents after the culture results [7]. This suggests that the clinical appearance of the patient and wound bed are perhaps more important than the results of any intraoperative cultures.

\section{Acknowledgments}

This article does not contain any studies with human participants performed by any of the authors. The patient gave the informed consent prior to being included into this case report. The author(s) received no financial support for the authorship or publication of this article.

\section{Author Disclosure Statement}

The authors declare no conflict of interest.

\section{References}

1. Paley D, Herzenberg JE. Intramedullary infections treated with antibiotic cement rods: preliminary results in nine cases. J Orthop Trauma 2002;16:723-729.

2. Hake ME, Young H, Hak DJ, et al. Local antibiotic therapy strategies in orthopaedic trauma: Practical tips and tricks and review of the literature. Injury 2015;46:1447-1456.

3. Thonse R, Conway JD. Antibiotic cement-coated nails for the treatment of infected nonunions and segmental bone defects. J Bone Joint Surg Am 2008;90(Suppl 4):163-174.

4. Masquelet AC, Begue T. The concept of induced membrane for reconstruction of long bone defects. Orthop Clin North Am 2010;41:27-37.

5. Dunbar RP. Treatment of infection after fracture fixation. Opinion: Retain stable implant and suppress infection until union. J Orthop Trauma 2007;21:503-505.

6. Kendall RW, Duncan CP, Smith JA, Ngui-Yen JH. Persistence of bacteria on antibiotic loaded acrylic depots. A reason for caution. Clin Orthop Relat Res 1996;329:273-280.

7. Moussa FW, Anglen JO, Gehrke JC, et al. The significance of positive cultures from orthopedic fixation devices in the absence of clinical infection. Am J Orthop 1997;26:617-620.

Address correspondence to: Dr. Tyler Snoap

Western Michigan University

Homer Stryker MD School of Medicine

Department of Orthopaedics

Western Michigan University

1000 Oakland Drive

Kalamazoo, MI 49008

E-mail: Tyler.Snoap@med.wmich.edu

Abbreviation Used

PICC $=$ peripherally inserted central catheter line

Cite this article as: Snoap T, Hart J, Roberts J (2017)

Successful bone grafting via masquelet technique around a locked antibiotic nail for tibial shaft fracture with polymicrobial infection. Surgical Infections Case Reports 2:1, 105-108, DOI: 10.1089/crsi.2017.0032 